\title{
Mutational landscape of thymic epithelial tumors in a Chinese population: insights into potential clinical implications
}

\author{
Hongbiao Wang ${ }^{1 \#}$, Xiaohua $\mathrm{Xu}^{2 \#}$, Lan Luo ${ }^{3}$, Chunbing Wang ${ }^{4}$, Zeyong Jiang ${ }^{5}$, Yingcheng Lin ${ }^{1}$ \\ ${ }^{1}$ Medical Oncology Session No.1, Cancer Hospital of Shantou University Medical College, Shantou, China; ${ }^{2}$ Cardiothoracic surgery department, \\ The Second Affiliated Hospital of Shantou University Medical College, Guangzhou, China; ${ }^{3}$ Department of Oncology, Integrated Hospital of \\ Traditional Chinese Medicine, Southern Medical University, Guangzhou, China; ${ }^{4}$ Medical Oncology Department, Cancer Hospital Chinese \\ Academy of Medical Science, Shenzhen, China; ${ }^{5}$ Department of Thoracic Surgery, Affiliated Cancer Hospital \& Institute of Guangzhou Medical \\ University, Guangzhou, China \\ Contributions: (I) Conception and design: Y Lin; (II) Administrative support: Y Lin, H Wang, X Xu; (III) Provision of study materials or patients: H \\ Wang, L Luo, C Wang, Z Jiang; (IV) Collection and assembly of data: H Wang, X Xu, L Luo, C Wang, Z Jiang; (V) Data analysis and interpretation: \\ H Wang, X Xu, L Luo, C Wang, Z Jiang, Y Lin; (VI) Manuscript writing: All authors; (VII) Final approval of manuscript: All authors. \\ "These authors contributed equally to this work. \\ Correspondence to: Dr. Yingcheng Lin. Medical Oncology Session No.1, Cancer Hospital of Shantou University Medical College, 7 Raoping Road, \\ Jinping District, Shantou 515041, China. Email: yclin@stu.edu.cn.
}

Background: Thymic epithelial tumors (TETs) are a heterogeneous group of rare malignancies which
may be devastating, difficult to treat, and for which treatment options are limited. Herein, we investigated
the comprehensive genomic alterations of TETs in a Chinese population for providing clinical management,
especially targeted therapy.

Methods: Comprehensive genomic profiling (CGP) was performed with DNA targeted sequencing of cancer-associated genes (CSYS) from a cohort of 40 Chinese TET patients. TMB was measured by an inhouse algorithm. MSI status was inferred based on the MANTIS (Microsatellite Analysis for Normal-Tumor InStability) score. The expression status of PD-L1 was estimated by immunohistochemistry.

Results: The mutational profiling of thymomas (Ts) and thymic neuroendocrine tumors (TNETs) showed scattered mutation distributions with no recurrently mutated genes. In contrast, thymic carcinomas (TCs) did show highly recurrent mutations including CDKN2A,CYLD, CDKN2B, and TP53. Among them, $C D K N 2 A$ and $C D K N 2 B$ mutations were the top potentially actionable alterations in TCs. PD-L1 expression was mainly present in Ts and TCs, and was predominant in males and smokers.

Conclusions: Our study provided a comprehensive genetic alteration view on the largest Chinese cohort of TETs to date. The results identified different genomic mutational profiles of Ts, TCs, and TNETs, and analyzed potential druggable biomarkers with clinical implications in Chinese TET patients, which provided the evidence for precision medicine of rare TET patients.

Keywords: Thymic epithelial tumors (TETs); thymomas (Ts); thymic carcinomas (TCs); thymic neuroendocrine tumors (TNETs); mutational landscape; actionable mutations

Submitted Jan 11, 2021. Accepted for publication Apr 20, 2021.

doi: $10.21037 / g s-21-157$

View this article at: http://dx.doi.org/10.21037/gs-21-157

\section{Introduction}

Thymic epithelial tumors (TETs) originate in the thymus and include thymomas (Ts), thymic carcinomas (TCs), and thymic neuroendocrine tumors (TNETs) $(1,2)$. Although
TETs are one of the rarest malignancies, they are the most common malignancies of the anterior mediastinum. The incidence of TETs is estimated to be 3.93 cases/million in China (3), but TETs are significantly rarer in North 
America, in which the incidence is 1.5 cases/million (4). This difference in incidence is of interest, as it may indicate the genetic differences between ethnicities (4).

According the criterion of World Health Organization (WHO), Ts are further classified into 6 different histological subtypes $(\mathrm{A}, \mathrm{AB}, \mathrm{B} 1, \mathrm{~B} 2$, and $\mathrm{B} 3$ of Ts, and $\mathrm{C}$ represents TC) (5). The subtypes are evaluated by morphology and lymphocyte/epithelial cell ratio. Although TCs are type C in the WHO classification, they are significantly different from Ts. TCs are rarer but much more aggressive. The 5 -year survival rate for Ts is approximately $90 \%(6-8)$, whereas it is approximately $55 \%$ for TCs (9-11).

The recommended curative treatment for localized TETs is complete surgical resection with or without postoperative radiation. However, there are little clinical data regarding second-line systemic therapy. For refractory or recurrent TETs, conventional chemotherapy has shown modest antitumor activity (12), while everolimus (13) for Ts and TCs, and sunitinib (14) and pembrolizumab (15) for TCs were accepted as second-line systemic therapies in the National Comprehensive Cancer Network (NCCN) guideline. However, due to the rarity of TETs, none of the targeted therapies or immunotherapies have been assessed in randomized phase 3 trials.

To enable discovery of novel targets and their potential clinical implications, we attempted to broadly and systematically investigate the comprehensive genomic alterations of TETs in a Chinese population to guide treatment decisions, especially for targeted therapy. Meanwhile, we also assessed the microsatellite stability (MSS) status, tumor mutation burden (TMB) value, and programmed death-ligand 1 (PD-L1) expression in TETs, which are predictive for immunotherapy.

We present the study in accordance with the MDAR reporting checklist (available at http://dx.doi.org/10.21037/ gs-21-157).

\section{Methods}

The study was conducted in accordance with the Declaration of Helsinki (as revised in 2013). The study was approved by the ethical committee of Cancer Hospital of Shantou University Medical College (No. 2020061) and informed consent was taken from all the patients.

\section{Participants}

This study included 40 Chinese TET patients whose tumor and matched blood specimens were randomly collected between 2014 and 2019 from multiple centers. Histological subtypes for each case were evaluated by pathologists in each hospital according to WHO criteria. The diagnosis of the slides was reviewed by independent pathologists. All participants were clearly informed, understood the contents of the study, and agreed to the data of the study being published. Informed written consent was provided by each participant.

\section{Sample preparation}

Formalin-fixed paraffin-embedded (FFPE) tissue samples and matched blood specimens of TET participants were collected from accredited clinical hospitals. The histologic sections were retrieved. It was ensured that the percentage of tumor cells for each sample was more than $20 \%$.

\section{Targeted next-generation sequencing (NGS) and genetic analysis}

Genomic DNA extraction (including FFPE tumor samples and matched white blood cells) and Cancer Sequencing YS panel (CSYS) was performed as previously described $(16,17)$. Briefly, 50-250 ng DNA was extracted for subsequent genetic analysis. Genetic alteration detection was performed with CSYS panel which covers all the coding exons of $450+$ cancer-related genes and selected introns of 39 genes that were frequently rearranged in solid tumors. Customized bioinformatics pipelines were applied for detection of single nucleotide variants (SNVs), short and long indels, copy number variations (CNVs), gene rearrangements, TMB, and microsatellite instability (MSI). All detected mutations were compared with our in-house database of genomic changes specific to clinical annotation.

\section{TMB calculation}

The TMB was estimated as previously described (17). Briefly, the total number of somatic mutations including coding SNVs and short indels were counted, and driver mutations and known germline alternations in the Single Nucleotide Polymorphism Database (dbSNP) were excluded. The TMB was calculated by dividing the total number of mutations counted by the size of the coding region.

\section{PD-L1 immunobistochemistry (IHC) staining assay}

The expression status of PD-L1 was estimated by IHC 
Table 1 Clinicopathological characteristics of 40 TET participants

\begin{tabular}{|c|c|}
\hline Characteristics & n (\%) \\
\hline Total number & 40 \\
\hline Age (years), median [range] & $50.8[26-74]$ \\
\hline \multicolumn{2}{|l|}{ Gender } \\
\hline Male & $26(65)$ \\
\hline Female & $14(35)$ \\
\hline \multicolumn{2}{|l|}{ Smoking status } \\
\hline Non-smoker & $16(40)$ \\
\hline Smoker and ex-smoker & $10(25)$ \\
\hline Undefined & $14(35)$ \\
\hline \multicolumn{2}{|l|}{ Stage } \\
\hline 1 & $4(10)$ \\
\hline II & $0(0)$ \\
\hline III & $6(15)$ \\
\hline IV & $15(37.5)$ \\
\hline Undefined & $16(37.5)$ \\
\hline \multicolumn{2}{|l|}{ Histological subgroup } \\
\hline \multicolumn{2}{|l|}{ Thymoma (total 21) } \\
\hline Type A & $1(2.5)$ \\
\hline Type AB & $3(7.5)$ \\
\hline Type B1 & $1(2.5)$ \\
\hline Type B2 & $6(15)$ \\
\hline Type B3 & $4(10)$ \\
\hline Combined B & $6(15)$ \\
\hline \multicolumn{2}{|l|}{ Thymic carcinoma (total 15) } \\
\hline Squamous cell carcinoma & $10(25)$ \\
\hline Adenocarcinoma, NOS & $1(2.5)$ \\
\hline Lymphoepithelioma-like carcinoma & $1(2.5)$ \\
\hline Unknown & $3(7.5)$ \\
\hline \multicolumn{2}{|l|}{ Thymic neuroendocrine tumor (total 4) } \\
\hline Carcinoid tumor & $2(5)$ \\
\hline Small-cell carcinoma & $1(2.5)$ \\
\hline Undefined & $1(2.5)$ \\
\hline Autoimmune disease (in thymomas) & $7(33.3)$ \\
\hline Myasthenia gravis & $6(28.6)$ \\
\hline Systemic lupus erythematosus & $1(2.5)$ \\
\hline
\end{tabular}

TET, thymic epithelial tumor. staining of FFPE tissue sections using anti-PD-L1 antibodies [clone 28-8; Cat\#ab205921; Abcam (Cambridge, UK) or clone 22C3; Cat\#M3653; Dako (Hovedstaden, Denmark)]. The specimen was considered to be PD-L1 positive if the staining cell percentage $\geq 1 \%$.

\section{Statistical analysis}

Statistical analyses were performed using SPSS version 22.0 (SPSS Inc., Chicago, IL, USA). Fisher's exact test was used to analyze significant differences. $\mathrm{P}<0.05$ was considered statistically significant.

\section{Results}

\section{Demographic and clinicopathological characteristics of participants}

To analyze the mutational features of TETs, a total of 40 patients were included in this study, including $21 \mathrm{Ts}$, 15 TCs, and 4 TNETs. Histological subtypes for each sample were evaluated by WHO criteria. The median age of participants at the time of sampling was 50.8 years (26-74 years), and $65 \%$ of participants were male. The demographic and clinicopathological characteristics of the 40 participants are shown in Table 1.

\section{Landscape of genetic alterations}

To investigate the distinctive genomic features of Chinese TETs, the 40 participants were subjected to a hybridization capture-based NGS panel (CSYS panel). Overall, 130 genetic alterations were identified in 84 genes. All participants had a signature of MSS except for 1 which was undefined. The average TMB was 2.285; approximately $1.75,3.22$, and 1.6 in Ts, TCs, and TNETs, respectively. The prevalence and distribution of genomic alterations are summarized in Figure 1.

The average number of mutations per participant was 3.25; approximately $1.43,5.73$, and 3.5 in Ts, TCs, and TNETs, respectively. Correlating with the lowest mutational prevalence, 4 out of 21 thymoma participants were identified with 0 mutations (case T03, T06, T08, and T19). Mutational analysis did not identify any recurrently mutated genes except for TAF1 and SETD 2 which occurred in 2 cases $(9.5 \%)$ in this subset. The scattered mutation distribution of Ts in a profiling map indicated the low recurrent frequency of the mutated genes. The 


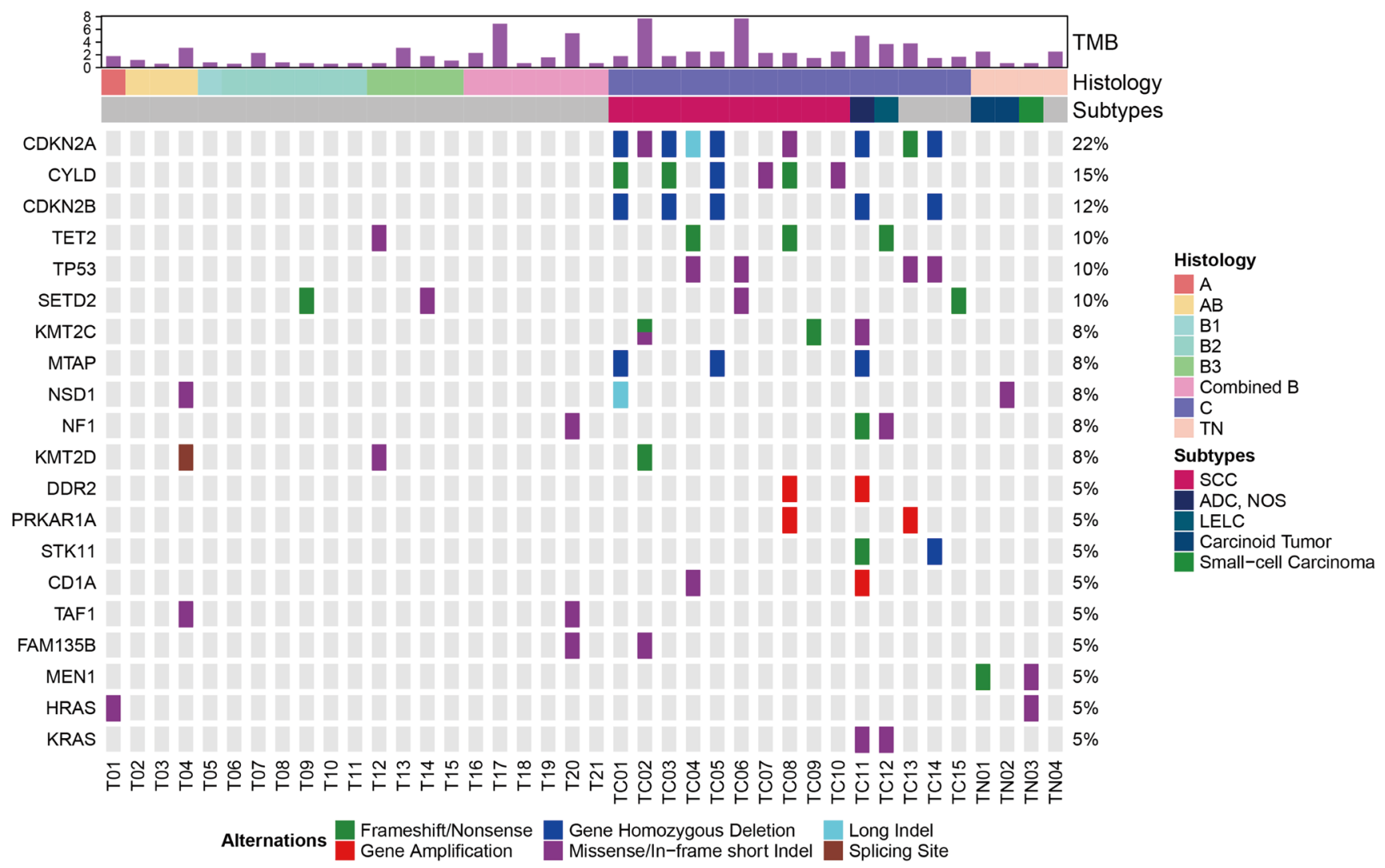

Figure 1 Molecular landscape of genetic alterations in all 40 TETs. TET, thymic epithelial tumor; T, thymoma; TC, thymic carcinoma; TNET, thymic neuroendocrine tumor.

most commonly mutated genes in TCs were $C D K N 2 A$ (9, 60\% in TC), CYLD (6, 40\%), CDKN2B (5, 33.3\%), and $\operatorname{TP} 53(4,26.7 \%)$. Notably, the majority of mutations of $C D K N 2 A$ were potential loss-of-function mutations, with the most significant gene deletions in 5 samples (case TC01, 03, 05, 11, and 14), missense mutations in 2 samples (case TC02 and 08), nonsense mutation in 1 sample (case TC13), and long deletion in exon 1 to intron 1 (case TC04). The genomic location of $C D K N 2 A$ is at chr9q21.3, where $C D K N 2 B$ and MTAP also locate. All 5 gene deletions of $C D K N 2 A$ were accompanied by $C D K N 2 B$ gene deletions, among which 3 cases harbored MTAP gene deletions (case TC01, 05, 11). TCs were characterized by gene deletions/amplifications, and 9 of the 15 samples had CNV of specified genes. For TNETs, we also observed scattered mutation distributions with no recurrently mutated genes identified except for MEN1. Case TN01 harbored 2 frameshift mutations in MEN1 (1 was somatic, the other was germline), and case TN03 harbored a somatic missense mutation in MEN1.
Given the prevalence and important role of MEN1 in neuroendocrine tumors, it was not surprising to observe the mutations in our subset.

\section{Predictive biomarkers with therapeutic perspective}

We explored the prevalence of actionable mutations of which targeted inhibitors have been encountered in clinical practice in TETs or other tumor types. Actionable mutations were based on databases including Oncology Knowledge Base (OncoKB, www.oncokb.org), Catalogue of Somatic Mutations in Cancer (COSMIC, cancer.sanger.ac.uk/cosmic), ClinicalTrials (cancer. sanger.ac.uk/cosmic/), and PubMed (www.ncbi.nlm.nih. gov/pubmed). Overall, 22 actionable mutations were identified in 8 genes. The tumor types with the highest proportion of actionable mutations were TCs (19, $86.4 \%)$, TNs $(2,9.1 \%)$, and Ts $(1,4.5 \%)$, sequentially. The most frequently recurrent actionable alterations included $C D K N 2 A(\mathrm{n}=9), \operatorname{CDKN} 2 B(\mathrm{n}=5), \operatorname{STK} 11(\mathrm{n}=2)$, 


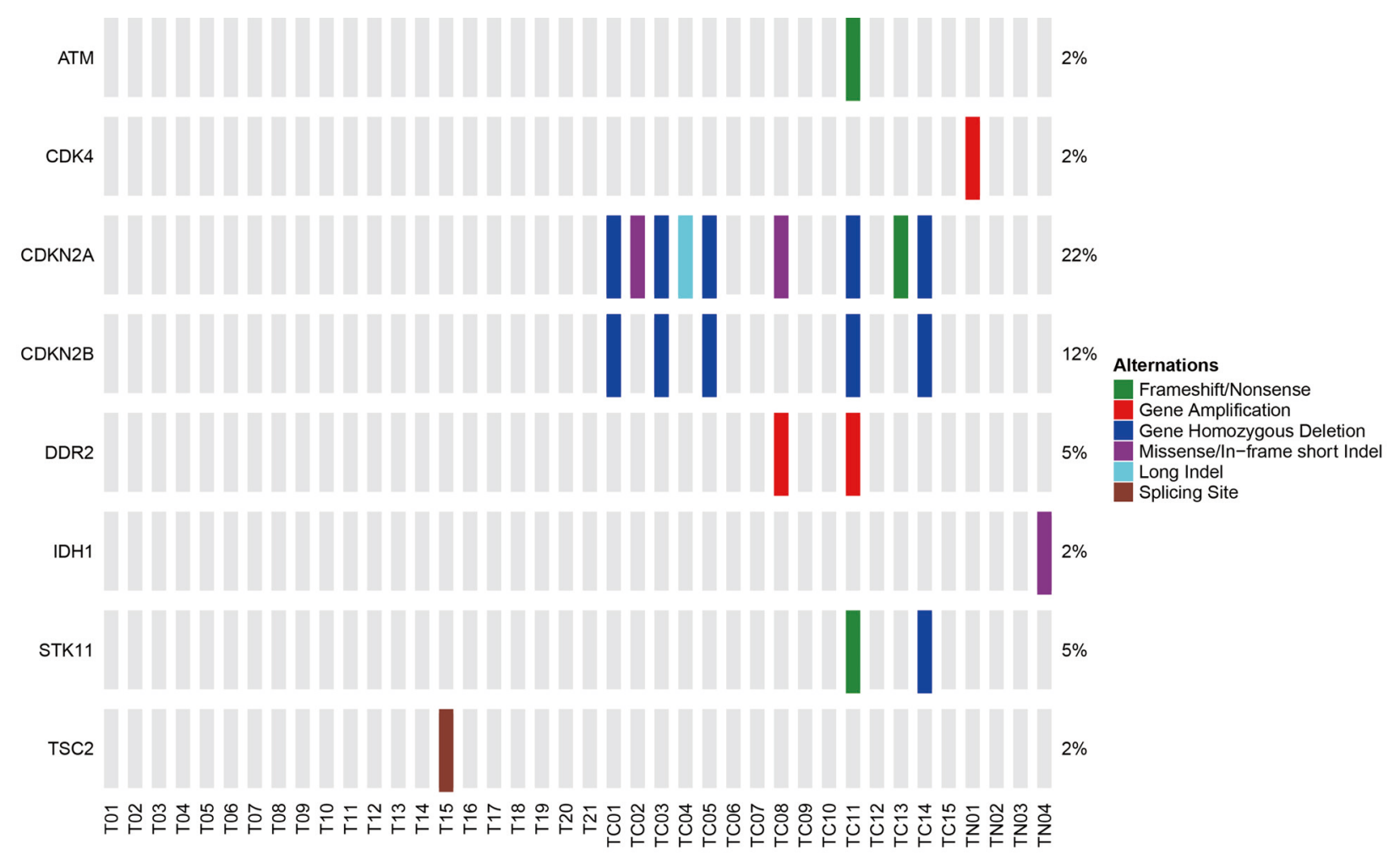

Figure 2 Actionable mutations identified in 40 TETs. TET, thymic epithelial tumor.

Table 2 Overall expression patterns of PD-L1

\begin{tabular}{lc}
\hline Clinical characteristic & $\begin{array}{c}\text { PD-L1 expression } \\
(\geq 1+\text { by IHC), } n(\%)\end{array}$ \\
\hline All $(n=24)$ & $12(50)$ \\
Gender & $9(60)$ \\
Male $(n=15)$ & $3(33.3)$ \\
Female $(n=9)$ & \\
Smoker & $4(40)$ \\
Non-smokers $(n=10)$ & $6(85.7)$ \\
Smoker and ex-smoker $(n=7)$ & \\
Histology & $6(54.6)$ \\
Thymomas $(n=11)$ & $6(54.6)$ \\
Thymic carcinomas $(n=11)$ & $0(0)$ \\
Thymic neuroendocrine tumors $(n=2)$ &
\end{tabular}

PD-L1, programmed death-ligand 1; IHC, immunohistochemistry.

and DDR2 $(\mathrm{n}=2)$ (Figure 2). Altogether, 12 participants (30\%), including 9 TCs, harbored at least 1 potentially actionable mutation.

\section{IHC detection of PD-L1}

Expression patterns of PD-L1 by IHC staining are depicted in Table 2. Inconsistent with a prior report (18), PDL1 expression was predominant in males and smokers. The expression of PD-L1 was present in $50 \%(12 / 24)$ of specimens examined, including $54.6 \%$ of Ts, $54.6 \%$ of TCs, and $0 \%$ of TNETs.

\section{Discussion}

The incidence of thymic tumors is under-estimated, and despite rarity, TETs are aggressive malignancies. With the increasing use of computed tomography (CT) screening for lung cancer, more early stage TETs are likely to be discovered. Moreover, the different incidence of TETs between Chinese and Western populations suggests that there may be a genetic component $(3,4)$. However, due to the rarity of TETs, there are few options for second-line systemic therapy. Therefore, precise therapeutic strategies with efficacy are needed.

Previous study showed that the most frequent mutated gene is GTF2I, and followed with HRAS, NRAS, and TP53 
in 117 TET patients, including $105 \mathrm{Ts}, 10 \mathrm{TCs}$, and 2 micorcodular thymomas, and most of patients were white race (19). Herein, we performed DNA targeted sequencing of cancer associated genes to analyze 40 Chinese TET patients. The results revealed that Ts, TCs, and TNETs are molecularly different malignancies. The mutational profiling of Ts and TNETs showed scattered mutation distributions with no recurrently mutated genes. In contrast, TCs did show highly recurrent mutations including $C D K N 2 A, C Y L D, C D K N 2 B$, and TP53. The small number of TETs are the limitation of these studies, which still need to be further confirmed.

C-KIT overexpression is reported to associate with the worse prognosis of TET, and activating mutations of c-Kit is the biomarker associated with response to imatinib $(20,21)$. Notably, we did not detect the c-KIT mutation in our TCs cohort for whom sunitinib is recommended. Previous studies reported the copy number aberrations of $C D K N 2 A / B$ (22) and loss of p16INK4A (23) are associated with worse prognosis in TCs. The genes $C D K N 2 A$ and $C D K N 2 B$ lie adjacently at chr9p21.3 which is frequently mutated or deleted across a wide variety of tumors. The $C D K N 2 A$ gene encodes p16INK4a and p14arf, while $C D K N 2 B$ encodes p15Ink4b. The encoded proteins bind to CDK4/6 thereby inhibiting its kinase activity. Gene deletions or mutations of $C D K N 2 A / 2 B$ could lead to the activation of CDK4/6 (24). Therefore, CDK inhibitors might target the $C D K N 2 A / 2 B$ gene deletions or mutations. The Food and Drug Administration (FDA) has approved the CDK4/6 inhibitors, palbociclib, ribociclib, and abemaciclib, to treat hormone receptor (HR)-positive and human epidermal growth factor receptor receptor 2 (HER2)-negative advanced or metastatic breast cancer. Several studies indicated $C D K N 2 A / 2 B$ gene deletions or mutations patients could benefit from CDK4/6 inhibitors (25-28). Previous clinical trial (NCT01291017) showed that nearly half of advanced non-small cell lung cancer patients with wild-type $R B$ and inactive $C D K N 2 A$, achieved stable disease for more than 4 months with the treatment of palbociclib.

Given pembrolizumab is active as second-line therapy in patients with TCs, we assessed the MSS status, TMB value, and PD-L1 expression in TETs. Overall, stable MSS and relatively low TMB value were observed in all 3 subtypes. Fortunately, $50 \%$ of Ts and TCs had PD-L1 expression which might be an indicative marker for immunotherapy in such patients.

\section{Conclusions}

In summary, we identified distinct genomic mutational profiles in Chinese TET patients with potentially actionable genetic alterations. The small number of patients is the limitation of this study. On account of the rarity and heterogeneity of TETs, it is critically important to initiate global or regional collaboration so as to accelerate the novel efficacy targeted- and immunotherapy in TETs.

\section{Acknowledgments}

Funding: None.

\section{Footnote}

Reporting Checklist: The authors have completed the MDAR reporting checklist. Available at http://dx.doi.org/10.21037/ gs-21-157

Data Sharing Statement: Available at http://dx.doi. org/10.21037/gs-21-157

Conflicts of Interest: All authors have completed the ICMJE uniform disclosure form (available at http://dx.doi. org/10.21037/gs-21-157). The authors have no conflicts of interest to declare.

Ethical Statement: The authors are accountable for all aspects of the work in ensuring that questions related to the accuracy or integrity of any part of the work are appropriately investigated and resolved. The study was conducted in accordance with the Declaration of Helsinki (as revised in 2013). The study was approved by the ethical committee of Cancer Hospital of Shantou University Medical College (No. 2020061) and informed consent was taken from all the patients.

Open Access Statement: This is an Open Access article distributed in accordance with the Creative Commons Attribution-NonCommercial-NoDerivs 4.0 International License (CC BY-NC-ND 4.0), which permits the noncommercial replication and distribution of the article with the strict proviso that no changes or edits are made and the original work is properly cited (including links to both the formal publication through the relevant DOI and the license). See: https://creativecommons.org/licenses/by-nc-nd/4.0/. 


\section{References}

1. Marx A, Chan JK, Coindre JM, et al. The 2015 World Health Organization Classification of Tumors of the Thymus: Continuity and Changes. J Thorac Oncol 2015;10:1383-95.

2. Travis WD, Brambilla E, Burke AP, et al. WHO Classification of Tumors of the Lung, Pleura, Thymus and Heart. Forth edition. WHO Classification of Tumors. Volume 7. Vol. 7: World Health Organization. 2015.

3. Fang W, Fu J, Shen Y, et al. Management of thymic tumors-consensus based on the Chinese Alliance for Research in Thymomas Multi-institutional retrospective studies. J Thorac Dis 2016;8:641-5.

4. Engels EA. Epidemiology of thymoma and associated malignancies. J Thorac Oncol 2010;5:S260-5.

5. Marx A. SP, Zettl A., et al. Thymomas. In: Travis WD, Brambilla E, Müller-Hermelink HK, Harris CC (eds). World Health Organization classification of tumours. Pathology and genetics of tumours of the lung, thymus and heart, vol. 7. Lyon, IARC Press. 2004:152-3.

6. Zhao Y, Shi J, Fan L, et al. Surgical treatment of thymoma: an 11-year experience with 761 patients. Eur J Cardiothorac Surg 2016;49:1144-9.

7. Huang J, Rizk NP, Travis WD, et al. Comparison of patterns of relapse in thymic carcinoma and thymoma. J Thorac Cardiovasc Surg 2009;138:26-31.

8. Masaoka A. Staging system of thymoma. J Thorac Oncol 2010;5:S304-12.

9. Litvak AM, Woo K, Hayes S, et al. Clinical characteristics and outcomes for patients with thymic carcinoma: evaluation of Masaoka staging. J Thorac Oncol 2014;9:1810-5.

10. Kondo K, Monden Y. Therapy for thymic epithelial tumors: a clinical study of 1,320 patients from Japan. Ann Thorac Surg 2003;76:878-84; discussion 884-5.

11. Eng TY, Fuller CD, Jagirdar J, et al. Thymic carcinoma: state of the art review. Int J Radiat Oncol Biol Phys 2004;59:654-64.

12. Rajan A, Carter CA, Berman A, et al. Cixutumumab for patients with recurrent or refractory advanced thymic epithelial tumours: a multicentre, open-label, phase 2 trial. Lancet Oncol 2014;15:191-200.

13. Zucali PA, De Pas T, Palmieri G, et al. Phase II Study of Everolimus in Patients With Thymoma and Thymic Carcinoma Previously Treated With Cisplatin-Based Chemotherapy. J Clin Oncol 2018;36:342-9.

14. Thomas A, Rajan A, Berman A, et al. Sunitinib in patients with chemotherapy-refractory thymoma and thymic carcinoma: an open-label phase 2 trial. Lancet Oncol 2015;16:177-86.

15. Cho J, Kim HS, Ku BM, et al. Pembrolizumab for Patients With Refractory or Relapsed Thymic Epithelial Tumor: An Open-Label Phase II Trial. J Clin Oncol 2019;37:2162-70.

16. Lin J, Shi J, Guo H, et al. Alterations in DNA Damage Repair Genes in Primary Liver Cancer. Clin Cancer Res 2019;25:4701-11.

17. Cao J, Chen L, Li H, et al. An Accurate and Comprehensive Clinical Sequencing Assay for Cancer Targeted and Immunotherapies. Oncologist 2019;24:e1294-302.

18. Owen D, Chu B, Lehman AM, et al. Expression Patterns, Prognostic Value, and Intratumoral Heterogeneity of PDL1 and PD-1 in Thymoma and Thymic Carcinoma. J Thorac Oncol 2018;13:1204-12.

19. Radovich M, Pickering CR, Felau I, et al. The Integrated Genomic Landscape of Thymic Epithelial Tumors. Cancer Cell 2018;33:244-58.

20. Terzi N, Yilmaz I, Batur S, et al. C-KIT mutation in thymic carcinomas. Pol J Pathol 2020;71:120-6.

21. Gao J, Dang Y, Sun N, Li J, Shen L. C-KIT mutations were closely associated with the response to Imatinib in Chinese advanced gastrointestinal stromal tumor patients. Med Oncol 2012;29:3039-45.

22. Petrini I, Meltzer PS, Zucali PA, et al. Copy number aberrations of BCL2 and CDKN2A/B identified by array-CGH in thymic epithelial tumors. Cell Death Dis 2012;3:e351.

23. Aesif SW, Aubry MC, Yi ES, et al. Loss of p16(INK4A) Expression and Homozygous CDKN2A Deletion Are Associated with Worse Outcome and Younger Age in Thymic Carcinomas. J Thorac Oncol 2017;12:860-71.

24. VanArsdale T, Boshoff C, Arndt KT, et al. Molecular Pathways: Targeting the Cyclin D-CDK4/6 Axis for Cancer Treatment. Clin Cancer Res 2015;21:2905-10.

25. Gao J, Adams RP, Swain SM. Does CDKN2A loss predict palbociclib benefit? Curr Oncol 2015;22:e498-501.

26. Infante JR, Cassier PA, Gerecitano JF, et al. A Phase I Study of the Cyclin-Dependent Kinase 4/6 Inhibitor Ribociclib (LEE011) in Patients with Advanced Solid Tumors and Lymphomas. Clin Cancer Res 2016;22:5696-705.

27. Patnaik A, Rosen LS, Tolaney SM, et al. Efficacy and Safety of Abemaciclib, an Inhibitor of CDK4 and 
CDK6, for Patients with Breast Cancer, Non-Small Cell Lung Cancer, and Other Solid Tumors. Cancer Discov 2016;6:740-53.

Cite this article as: Wang $\mathrm{H}, \mathrm{Xu} \mathrm{X}$, Luo L, Wang C, Jiang Z, Lin Y. Mutational landscape of thymic epithelial tumors in a Chinese population: insights into potential clinical implications. Gland Surg 2021;10(4):1410-1417. doi: 10.21037/gs-21-157
28. Sherr CJ, Beach D, Shapiro GI. Targeting CDK4 and CDK6: From Discovery to Therapy. Cancer Discov 2016;6:353-67. 